\title{
Tetralogy of Fallot Complicated With Preeclampsia After Three Cardiac Operations
}

\author{
Mehmet Kulhan ${ }^{1}$, Nur Gözde Kulhan ${ }^{2}$, Ümit Nayk1 ${ }^{3}$, Cenk Nayk1 ${ }^{4}$,Paşa Uluğ $\breve{g}^{5}$, \\ Nahit $\mathrm{Ata}^{6}$, Hülya Toklucu

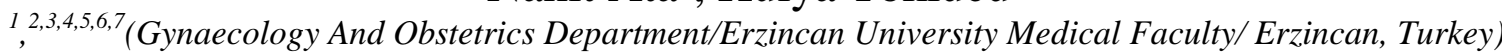

\begin{abstract}
Tetralogy of Fallot (ToF) is the most common form of cyanotic congenital heart diseases and without corrective surgery, natural survival rate into the fourth decade was only about $3 \%$. In pregnant patients with repaired ToF, pregnancy-related physiological changes is more difficult to tolerate compared to healthy pregnant woman. ToF rarely be associated with preeclampsia. Discussed below a case of a 20 year old woman with repaired ToF Complicated with Preeclampsia After Three Cardiac Operations.
\end{abstract}

Keywords: Obstetric outcomes, Pregnancy, Tetralogy of Fallot, Preeclampsia

\section{Introduction}

Tetralogy of Fallot $(\mathrm{ToF})$ is a syndrome of abnormalities due to maldevelopment of the truncus arteriosus characterized by right ventricular outflow tract obstruction, ventricular septal defect (VSD), overriding aorta and right ventricular hypertrophy. ToF is the most common cyanotic congenital heart disease, and it was among the first to be successfully surgically palliated by Blalock and Taussig in 1945 and subsequently physiologically repaired. Therefore, a significant number of adults with congenital heart disease have repaired ToF [1]. Pregnancy is not recommended in patients with unrepaired ToF [2]. However, after corrective surgery, women with ToF generally have good maternal and infant outcomes if they do not have severe hemodynamic abnormalities before pregnancy [3]. Preeclampsia is a multi-system disorder characterized by the new onset of hypertension and proteinuria or end-organ dysfunction or both in the last half of pregnancy. ToF rarely be associated with preeclampsia. Patients with significant cyanosis at birth often had palliative Blalock-Taussing shunts in infancy using the subclavian artery to connect the systemic circulation with the pulmonary circulation, thus increasing pulmonary blood flow and reducing cyanosis. As a consequence, blood pressures in the arm supplied by the transected artery may not be reflective of aortic pressure. Patients should be asked if one arm is unreliable for blood pressure measurements, or examined to look for evidence of a thoracotomy scar resulting from the Blalock-Taussig shunt. In this case report, we present a 20 year old woman with repaired ToF Complicated with Preeclampsia After Three Cardiac Operations.

\section{Case Report}

A 20-years old young female from Afghanistan is a known case of Congenital Cyanotic Heart Disease ToF was admitted at 34 weeks gestation with suspected preeclampsia. She had irregular antenatal visits, which revealed no abnormality. It was learned that she was operated for ToF three times in the personal and family history of the case. She is 7 th in birth order, born at full term by normal vaginal delivery. She was apparently alright till the age 7 years when she was noticed to have bluish discoloration of skin and mucous membrane during exertion. She was then taken to a local practitioner who suspected her to have congenital cyanotic heart disease. Subsequently she was seen by local cardiologist and she was evaluated thoroughly including an echo which revealed ToF, VSD, severe pulmoner stenosis (PS). Parents were advised for early cardiac surgery. However, surgery was delayed till 14 years of age due to various reasons. There is no progressive cyanosis and easy fatigability. At 14 years of age, she underwent Blalock-Taussig shunt surgery in Afghanistan. Subsequently she also underwent central shunt as the previos shunt was got blocked. Thereafter her symptoms have improved till recently when she again started to have progressively increasing cyanosis, and easy fatigability so she underwent third operation. She was admitted for pregnancy checks about 2 years after the third operation in Turkey. She had a 34 weeks healthy pregnancy. She had 1+ proteinuria on dipstick and a Blood Pressure (BP) of 110/70 mmHg. Her $24 \mathrm{~h}$ proteinuria was $300 \mathrm{mg} / \mathrm{dL}$. Since then, she had been under scheduled follow up with clinical and echocardiographic examination, and she was in New York Heart Association (NYHA) functional class I to II with good cardiac function. On clinical examination the patient was pink (saturation 96\%, on room air) and well with $3+$ peripheral oedema. She had a normal first heart sound, single second, and a very soft ejection systolic heart murmur at the upper left sternal edge $1 / 6$ with no diastolic murmur. She was discharged 2 days later with a diagnosis of mild preeclampsia, to be followed as an outpatient. Four days later, she was readmitted with a BP of 130/100 mmHg measured at home. On readmission, her BP 
ranged between 120/90 and 140/100 $\mathrm{mmHg}$, with most values below 140/90 mmHg. She had 4+ proteinuria, 3+ peripheral oedema, a non- reactive non-stress test, and normal amniotic fluid volume and Doppler indexes. Laboratory findings were remarkable (hemoglobin $11.3 \mathrm{mg} / \mathrm{dL}$, hematocrit: $32.8 \%$, thrombocyte: $100000 / \mathrm{mm}^{3}$, glucose: $97 \mathrm{mg} / \mathrm{dL}$, creatinine: 1,6, ALT: $11 \mathrm{U} / \mathrm{L}$, AST: $47 \mathrm{U} / \mathrm{L}, \mathrm{LDH}: 519 \mathrm{U} / \mathrm{L}$ ), with a 4+of proteinuria in the urinalysis. She had no prodromes suggestive of hypertensive disease. During fetal monitorization, late decelerations were observed and delivery was performed via caesarean section. An emergency Cesarean delivery was performed for fetal distress and severe preeclampsia. A male fetus weighing $2450 \mathrm{~g}$ was delivered with APGAR scores of 7 and 8 at 1 and 5 min, respectively. No congenital cardiac anomaly was identified in infant. The patient was consulted to the cardiology department for further evaluation. There is no other adverse maternal cardiovascular events in early postpartum period. The patient received $3000 \mathrm{cc}$ intravenous fluids with $40 \mathrm{IU}$ oxitosin in postpartum period but urine output not followed and anuria detected and peripheral oedema increased. The patient had chief complaint of worsening difficulty of breathing since 4 hours after delivery so the patient was reconsulted to the cardiology department and right ventricular overload findings were observed. Although the using of furosemide is contraindicated in preeclampsia, furosemide infusion was started to the patient and fluid infusion was stopped. After this treatment, $2500 \mathrm{cc}$ diuresis was observed and respiratory distress and peripheral edema decreased in 8 hours. Three days later, she became normotensive and her complaints resolved. She was discharged on the 4 th postoperative day.

\section{Discussion}

$\mathrm{ToF}$ is the most common cyanotic congenital heart defect and comprises $75 \%$ of cyanotic cardiac diseases[4]. Most of the patients underwent a surgical correction operation in the early stages of their life. Fertility and life expectancy reduced in patients who don't underwent any surgical operations [5]. Pregnancy is well tolerated in patients with normal functional status after surgical correction. Uncorrected ToF reveal serious risks incluiding maternal mortality in pregnancy. Previous pulmonary hypertension is a serious problem. In addition; increased cardiac output leads to increased venous return to the hypertrophic right ventricle. These changes increases the right-to-left shunt with reduced vascular resistance. This leads to further cyanosis, a compensatory rise in hematocrit, and a corresponding decrease in arterial oxygen saturation and risk of cardiac failure will be increased in these cases[6]. A poor prognosis exists for patients whose shunting is of such a degree as to result in a hematocrit $60 \%$ or more or an arterial oxygen saturation of less than $80 \%$ [7]. In ToF cases pulmonary artery abnormalities, particularly in the presence of pulmonary regurgitation, may adversely affect augmentation of maternal cardiac output and this may result in depressed placental blood flow and, subsequently, intrauterine growth retardation [8]. Classically, corrected ToF is considered a low-risk lesion in terms of both maternal and fetal outcomes. Seven percent of the patients with repaired ToF developed adverse cardiovascular events, including supraventricular tachycardia, heart failure, pulmonary embolism and progression of right ventricular dysfunction during pregnancy [9]. In our case, the patient developed mild right ventricular dilatation and heart failure, which were reversed after delivery. Children of mothers with ToF are more likely to have congenital heart disease, with a reported incidence of $3 \%$. In addition, it is believed that $15 \%$ of patients with ToF have Di George syndrome, which is inherited with autosomal dominant pattern [9].

After surgical correction is only restricted to the activity in order to protect from fatigue. Termination of pregnancy is recommended for patients with uncorrected ToF. Hematocrit should be monitored. Successful cardiac surgery improves fertility and reduces the maternal and fetal risk of pregnancy in women with congenital heart disease. Decisions about timing and mode of delivery should be reached after an interdisciplinary discussion that also involves the patient. Vaginal delivery has a lower risk of complications for both the mother and fetus [10]. In our case, An emergency Cesarean delivery was performed for fetal distress and severe preeclampsia. The American Heart Association guidelines do not recommend antibiotic prophylaxis in vaginal or caesarean delivery [11], but in practice most centres give prophylaxis, as we did in our patient. Oxytocin and ergometrine have unpredictable effects on the haemodynamic status and should be avoided[12] bu we did in our case. Close observation and continuous haemodynamic monitoring are mandatory during the delivery and up to one week postpartum. No anticoagulation was administered to our patient during the pregnancy or puerperium.

"The authors declare that they have no conflict of interest."

"All procedures performed in studies involving human participants were in accordance with the ethical standards of the institutional and/or national research committee and with the 1964 Helsinki declaration and its later amendments or comparable ethical standards."

"This article does not contain any studies with human participants or animals performed by any of the authors."

\section{References}

[1]. Downing TE, Kim YY. Tetralogy of Fallot: General Principles of Management. Cardiol Clin. 2015 Nov;33(4):531-41, vii-viii. doi: 10.1016/j.ccl.2015.07.002. Epub 2015 Aug 29. Review. 
[2]. Warnes CA, Williams RG, Bashore TM, et al. ACC/AHA 2008 guidelines for the management of adults with congenital heart disease: a report of the American College of Cardiology/American Heart Association Task Force on Practice Guidelines (Writing Committee to Develop Guidelines on the Management of Adults With Congenital Heart Disease). Developed in Collaboration With the American Society of Echocardiography, Heart Rhythm Society, International Society for Adult Congenital Heart Disease, Society for Cardiovascular Angiography and Interventions, and Society of Thoracic Surgeons. J Am Coll Cardiol $2008 ; 52:$ e143.

[3]. Engelfriet P, Boersma E, Oechslin E, et al. The spectrum of adult congenital heart disease in Europe: morbidity and mortality in a 5 year follow-up period. The Euro Heart Survey on adult congenital heart disease. Eur Heart J 2005; 26:2325.

[4]. Koplay M, Erol C, Uysal E, Paksoy Y. Magnetic resonance imaging findings of absent pulmonary valve syndrome associated with tetralogy of fallot. Wien Klin Wochenschr. 2011 Sep;123(17-18):524-5. doi: 10.1007/s00508-011-0036-6.

[5]. Siu SC, Sermer M, Colman JM et al. Prospective multicenter study of pregnancy outcomes in women with heart disease. Circulation. 2001 Jul 31;104(5):515-21.

[6]. Pitkin RM, Perloff JK, Koos BJ, Beall MH. Pregnancy and congenital heart disease. Ann Intern Med. 1990 Mar 15;112(6):445-54.

[7]. Davies GAL, Herbert WNP. Congenital Heart Disease in Pregnancy. J Obstet Gynaecol Can 2007;29(5):409-14.

[8]. Veldtman GR, Connolly HM, Grogan M, Ammash NM, Warnes CA. Outcomes of pregnancy in women withtetralogy of fallot. J Am Coll Cardiol 2004;44;174-180.

[9]. Chamaidi A, Gatzoulis MA. Heart disease and pregnancy. Hellenic J Cardiol. 2006; 47: 275-291

[10]. Uebing A, Steer PJ, Yentis SM, Gatzoulis MA. Pregnancy and congenital heart disease. BMJ. 2006; 332: 401-406.

[11]. Dajani AS, Taubert KA, Wilson W, et al. Prevention of bac-terial endocarditis. Recommendations by the American Heart Association. Circulation. 1997; 96: 358-366.

[12]. Lupton M, Oteng-Ntim E, Ayida G, Steer PJ. Cardiac disease in pregnancy. Curr Opin Obstet Gynecol. $2002 ; 14: 137-143$. 